\title{
A Modified Range Migration Algorithm for FMCW SAR Signal Processing
}

\author{
Yake Li ${ }^{*}$, Siu O'Young
}

Faculty of Engineering and Applied Science, Memorial University of Newfoundland, St. John's, Canada; Email: y11323@mun.ca1

Abstract: The range migration algorithm (RMA) is an accurate imaging method for processing synthetic aperture radar (SAR) signals. However, this algorithm requires a big amount of computation when performing Stolt mapping. In high squint and wide beamwidth imaging, this operation also requires big memory size to store the result spectrum after Stolt mapping because the spectrum will be significantly expanded. A modified Stolt mapping that does not expand the signal spectrum while still maintains the processing accuracy is proposed in this paper to improve the efficiency of the RMA when processing frequency modulated continuous wave (FMCW) SAR signals. The modified RMA has roughly the same computational load and required the same memory size as the range Doppler algorithm (RDA) when processing FMCW SAR data. In extreme cases when the original spectrum is significantly modified by the Stolt mapping, the modified RMA achieves better focusing quality than the traditional RMA. Simulation and real data are used to verify the performance of the proposed RMA.

Keywords: FMCW SAR; Range migration algorithm

\section{Introduction}

Frequency modulated continuous wave (FMCW) synthetic aperture radar (SAR) has been developed and experimented extensively in recent years ${ }^{[1-7]}$. A common technique employed in these systems is the dechirp-on-receive demodulation that can reduce the requirements for radar receiver. The intermediate frequency (IF) signal after dechirp-on-receive does not related to the transmitted signal bandwidth, which allows FMCW SAR to achieve high resolution without a complex analog to digital convertor (ADC) system. Another difference of FMCW SAR from pulsed SAR is the longer pulse length, which allows the Doppler shift inside the pulse to be measurable and compensable. The major pulsed SAR processing algorithms have been modified and applied in FMCW SAR signal processing $^{[8-11]}$.
The $\mathrm{RMA}^{[12,13]}$ is an accurate algorithm that is especially effective to process large squint and wide beamwidth SAR signals. Motion compensation ${ }^{[14]}$ and autofocus ${ }^{[15,16]}$ has been integrated in RMA for image quality improvement. The extensions of RMA have integrated this algorithm with motion compensation $^{[14]}$ or autofocus ${ }^{[15,16]}$. However, the computational load of RMA is considerably high ${ }^{[10]}$, especially when the Stolt mapping has to extensively expand the target spectrum in wide beamwidth or high squint imagery. Moreover, the expansion of the spectrum size requires more memory capability, which limits the application of RMA in real time processing.

Dechirp-on-receive compresses the range modulation before ADC. According to the time-frequency relationship of a chirp signal, this operation has equivalently transform the IF data to its range frequency domain ${ }^{[17]}$.

Copyright (C) 2019 Yake Li et al.

doi: $10.18282 /$ rs.v8i1.517

This is an open-access article distributed under the terms of the Creative Commons Attribution Unported License

(http://creativecommons.org/licenses/by-nc/4.0/), which permits unrestricted use, distribution, and reproduction in any medium, provided the original work is properly cited 
This makes it convenient for the application of RAM because only one azimuth Fourier Transform (FT) is needed to obtain the two di-mensional spectrum. Also, the in-pulse Doppler shift ${ }^{[8]}$ induced by the continuous moving of the aircraft corresponds to an azimuth-frequency dependent phase term in the two dimensional frequency domain, which could be easily removed by the reference function multiplication (RFM) in RMA.

Except the convenience in application, dechirp-on-receive can also improve the efficiency of the Stolt mapping by exploring the characteristic of the IF signal, which is the content of this paper. The modified Stolt mapping has two differences from the traditional one. The first difference is that the mapping equation is changed to eliminate the parallel shifting of the spectrum. The second difference is that the range of the new variable after Stolt mapping can be limited without losing accuracy. The modified RMA has roughly the same computational load and the same memory requirements as the range Doppler algorithm (RDA), but can handle more general SAR imaging configurations. This makes the proposed RMA an efficient and practical algorithm to process FMCW SAR signals in either real-time or post processing.
This paper is organized as follows. Section 2 discusses the range resolution of FMCW radar. The result is crucial to demonstrate the range resolution of the modified Stolt mapping. Section 3 derives the spectral model for FMCW SAR signal and introduced the modified RMA. Section 4 shows the flow diagram of the RMA, which is then compared with the RDA flow chart. Simulation and actual data are used in Section 5 to verify proposed algorithm.

\section{Range resolution of the IF signal in FMCW SAR}

A sawtooth linear frequency modulated (LFM) signal (shown in Figure 1) can be expressed as

$$
s_{T}(t)=\operatorname{rect}\left(\frac{t}{T}\right) \exp \left(j \pi k t^{2}+j 2 \pi f_{0} t\right)
$$

where

$$
\operatorname{rect}\left(\frac{t}{T}\right)=\left\{\begin{array}{lr}
1 & |t| \leq T / 2 \\
0 & \text { others }
\end{array}\right.
$$

is the gate function. $\mathrm{T}$ is the period of the signal, $f_{0}$ is the centre frequency, $\mathrm{Bw}$ is bandwidth and $k=B w / T$ is the frequency modulation (FM) rate. The returned signal can be expressed as

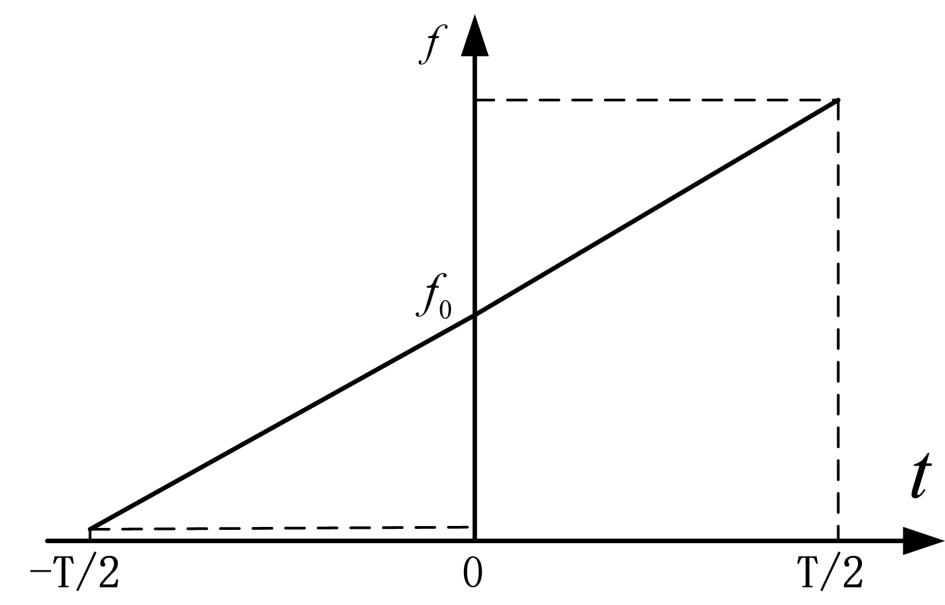

Figure 1. Sawtooth LFM signal.

$s_{R}(t)=\operatorname{rect}\left(\frac{t}{T}\right) \exp \left[j \pi k(t-\tau)^{2}+j 2 \pi f_{0}(t-\tau)\right]$

where $\tau=2 R_{0} / c$ is the two-way time delay, $R_{0}$ is the distance between the radar and the target, $c$ is the speed of light. The amplitude of the returned signal is assumed to be one without loss of generality. In real case, $R_{0}$ is normally no more than several kilometers for
FMCW SAR, hence the time delay $\tau$ is in the order of tens of microseconds. This delay will slightly decrease the frequency beating length between the reference signal and the received signal, which will lower the resolution. Since the delay is very small compared with $T$ (normally in the order of milliseconds), its effect on the resolution could be neglected. Therefore, it is neglected in the rectangular function in (3). However, $\tau$ cannot be neglected in the phase because its coefficient 
in the phase is sufficiently large to make the two-way time delay a significant contribution to the change of the phase. By measuring the changes in phase, the linear frequency modulated (LFM) signal could measure the distance of the target.

The IF signal is the result of multiplying the received signal (3) with the transmitted signal (1), which is

$s_{I F}(t)=\operatorname{rect}\left(\frac{t}{T}\right) \exp \left(j 2 \pi k \tau t-j \pi k \tau^{2}+j 2 \pi f_{0} \tau\right)$

This is the so called dechirp-on-receive demodulation. The FT of (4) is used to measure the range of the target, which is

$$
\begin{gathered}
S_{I F}(f)=T \operatorname{sinc}[T(f-k \tau)] e^{j 2 \pi f_{0} \tau} e^{-j \pi k \tau^{2}} \\
\operatorname{sinc}(\mathrm{x})=\frac{\sin (\pi x)}{\pi x}
\end{gathered}
$$

Equation (5) shows that the FT result of the IF signal is a sinc signal at $f=k \tau \mathrm{Hz}$.

After ADC sampling, the $3 \mathrm{~dB}$ width of the sinc function shown in (5) in digital frequency domain is ${ }^{[14]}$

$$
\frac{1}{T}(H z)=1(\text { sample })
$$

The center of the sinc function is at

$$
\frac{k \tau}{\Delta f}=\frac{(B w / T) \cdot\left(2 R_{0} / c\right)}{(1 / T)}=\frac{R_{0}}{(c /(2 \cdot B w))}=\frac{R_{0}}{\rho_{s}} \quad \text { (samples) }
$$

where $\rho_{s}=C /(2 \cdot B w)$ is the range resolution of the transmitted signal.

In a pulsed radar system, orthogonal demodulation is normally used to demodulate the received signal instead of the dechirp-on-receive technique. Then the absolute value of the pulse compression results in an orthogonal demodulation radar system when transmitting the signal shown by (1) will be ${ }^{[18]}$

$$
s_{1}(t)=T \sin c\left[k T\left(t-\frac{2 R_{0}}{c}\right)\right]
$$

whose $3 \mathrm{~dB}$ width is $1 / k T$. Compare (8) with (5), it can be seen that the expression of the resolution for the IF signal is different in FMCW SAR from that in pulse SAR. This is not surprising because the resolution is realized in frequency domain in dechirp-on-receive radar while in orthogonal demodulation system, the resolution is expressed in time domain. Therefore, the implementation of the traditional Stolt mapping in pulse SAR signal processing can be made more efficient in FMCW SAR signal processing.

\section{The modified range migration algorithm}

\subsection{The equivalent two dimensional spectrum of FMCW SAR}

Assuming the transmitted signal is

$$
s_{T}(t, \eta)=\operatorname{rect}_{r}\left(\frac{t}{T}\right) \operatorname{rect}_{a}\left(\frac{\eta-\eta_{c}}{T_{a}}\right) e^{j \pi k t^{2}} e^{j 2 \pi f_{0} t}
$$

where $t$ is range time (fast time) and $\eta$ is azimuth time (slow time), $\eta_{c}$ is the zero azimuth Doppler time. The azimuth envelop is assumed to have the rectangular shape, though its precise form is similar to the mainlobe of a sinc function ${ }^{[18]} . T_{a}$ is the synthetic aperture time. The amplitude is assumed to be 1 without loss of generality. $k$ is the FM rate and $f_{0}$ is the carrier frequency. Assuming the SAR operates in broadside stripmap mode, the received signal ${ }_{\text {is }} s_{R}(t, \eta)=\sigma\left(R_{0}, \eta_{c}\right) \operatorname{rect}_{r}\left(\frac{t}{T}\right) \operatorname{rect}_{a}\left(\frac{\eta-\eta_{c}}{T_{a}}\right)$

$$
e^{j \pi k(t-\tau)^{2}} e^{j 2 \pi f_{0}(t-\tau)}
$$

where

$$
\tau=\frac{2 R(\eta)}{c}=\frac{2}{c} \sqrt{R_{0}^{2}+v^{2}\left(\eta+t-\eta_{c}\right)^{2}}
$$

$\sigma\left(R_{0}, \eta_{c}\right)$ is the reflection coefficient for the target at $\left(R_{0}, \eta_{c}\right)$. A more accurate expression for the two-way delay time can be found $\mathrm{in}^{[11]}$. The expression of (11) is accurate enough in normal airborne situations ${ }^{[10]}$.

The IF signal is the multiplication of (10) with the conjugate of (9), which is

$$
\begin{gathered}
s_{I F}(t, \eta)=\sigma\left(R_{0}, \eta_{c}\right) \operatorname{rect}_{r}\left(\frac{t}{T}\right) \operatorname{rect}_{a}\left(\frac{\eta-\eta_{c}}{T_{a}}\right) \\
e^{j 2 \pi f_{0} \tau} e^{j 2 \pi k \tau t} e^{-j \pi k \tau^{2}}
\end{gathered}
$$


The last exponential term $e^{-j \pi k \tau^{2}}$ in (12) is the residual video phase (RVP), and can be compensated before the start of image formation. Literature $^{[17]}$ gives a method to remove the RVP term. In the following derivation, this term is neglected.

Because the dechirp-on-receive has readily transformed the signal to its equivalent range frequency domain, only one azimuth FT is needed to generate the equivalent two dimensional frequency expression, which is

$$
\begin{gathered}
S\left(t, f_{\eta}\right)= \\
\sigma\left(R_{0}, \eta_{c}\right) \operatorname{rect}\left(\frac{t}{T}\right) \int \operatorname{rect}_{a}\left(\frac{\eta-\eta_{c}}{T_{a}}\right) \\
e^{j 2 \pi f_{0} \tau} e^{j 2 \pi k \tau t} e^{-j 2 \pi f_{\eta} \eta} d \eta
\end{gathered}
$$

As can be seen, the signal is actually in the range-time azimuth-frequency domain. The reason it is called equivalent two dimensional frequency domain is because the expression in (13) actually has the characteristic of the two dimensional frequency domain due to dechirp-on-receive ${ }^{[17]}$. After using POSP (principle of stationary phase $)^{[17]}$, the equivalent two-dimensional spectrum of the IF signal can be expressed by

$$
S\left(t, f_{\eta}\right)=\sigma\left(R_{0}, \eta_{c}\right) \operatorname{rect}\left(\frac{t}{T}\right) \operatorname{rect}_{a}\left(\frac{f_{\eta}}{B w_{a}}\right) \exp \left(j \Phi\left(t, f_{\eta}\right)\right)
$$

where

$$
\Phi\left(t, f_{\eta}\right)=\frac{4 \pi R_{0}}{c} \sqrt{\left(f_{0}+k t\right)^{2}-\frac{c^{2} f_{\eta}^{2}}{4 v^{2}}}+2 \pi f_{\eta} t-2 \pi f_{\eta} \eta_{c}
$$

and $B w_{a}$ is the azimuth bandwidth. It is an equivalent 'two-dimensional' spectrum because the range direction is actually the range time but not frequency. However, as the signal in range-time of FMCW SAR has the same form and properties as that in range-frequency of the pulsed radar, (15) is named equivalent two-dimensional spectrum in this paper.

Taylor expansion can be used to approximate the square root in (15) for better understanding the modified Stolt mapping. By using Taylor expansion, we have

$$
\begin{aligned}
& \Phi\left(t, f_{\eta}\right)=\frac{4 \pi R_{0}}{c}\left[f_{0} D\left(f_{\eta}, v\right)+\frac{k}{D\left(f_{\eta}, v\right)} t-\right. \\
& \left.\frac{k^{2} c^{2} f_{\eta}^{2}}{8 v^{2} f_{0}^{2} D^{3}\left(f_{\eta}, v\right)} t^{2}+o\left(t^{2}\right)\right]-2 \pi f_{\eta} t-2 \pi f_{\eta} \eta_{c}
\end{aligned}
$$

where

$$
D\left(f_{\eta}, v\right)=\sqrt{1-\frac{c^{2} f_{\eta}^{2}}{4 v^{2} f_{0}^{2}}}
$$

is the cosine of the instantaneous incidence angle of the radar. $o\left(t^{2}\right)$ of (16) is the higher order terms in Taylor expansion. The terms in the square bracket represents the Taylor expansion of the square root. The traditional Stolt mapping tries to correct all the terms in the square brackets to finish azimuth compression, range cell migration correction (RCMC), SRC (second range compression) and higher order terms correction.

\subsection{The modified RMA}

\subsubsection{Reference function multiplication}

The first step of the modified RMA is to multiply the reference function multiplication (RFM),

$$
\exp \left(j \Phi_{1}\left(t, f_{\eta}\right)\right)=\exp \left(-j \frac{4 \pi R_{\text {ref }}}{c} \sqrt{\left(f_{0}+k t\right)^{2}-\frac{c^{2} f_{\eta}^{2}}{4 v^{2}}}+j 2 \pi f_{\eta} t\right)
$$

This operation focuses the points in the reference range. The last term of (18) is to remove the in-pulse Doppler effect. The expression after RFM is

$$
\begin{gathered}
S_{1}\left(t, f_{\eta}\right)=\sigma\left(R_{0}, \eta_{c}\right) \operatorname{rect}\left(\frac{t}{T}\right) \operatorname{rect}_{a}\left(\frac{f_{\eta}}{B w_{a}}\right) \exp \\
\left(j \frac{4 \pi \Delta R}{c} \sqrt{\left.\left(f_{0}+k t\right)^{2}-\frac{c^{2} f_{\eta}^{2}}{4 v_{r e f}^{2}}-j 2 \pi f_{\eta} \eta_{c}\right)}\right.
\end{gathered}
$$

where $\Delta R=R_{0}-R_{\text {ref }}$.

\subsubsection{Modified stolt mapping}

The second step of the modified RMA is the modified Stolt mapping, which is applied to the square root of the phase in (19). Before introducing the modified Stolt mapping, the traditional Stolt mapping is analysed and the problems of its application in FMCW 
SAR processing is addressed. The traditional Stolt mapping is

$$
\sqrt{\left(f_{0}+k t\right)^{2}-\frac{c^{2} f_{\eta}^{2}}{4 v_{r e f}^{2}}}=f_{0}+k t_{1}
$$

where $t_{1}$ is the new time variable. This step is actually a change of variables, which corresponds to a mapping of the original time variable. For simplicity, we only use the first two term in the square bracket of (16) to represent the square root of (20). The higher order terms will also be corrected by the Stolt mapping. However, as they have no effect in the following derivation, they are neglected in the equations. Then we obtain the form of the variable change,

$$
t_{1}=\frac{f_{0}}{k}\left(D\left(f_{\eta}, v\right)-1\right)+\frac{t}{D\left(f_{\eta}, v\right)}
$$

The first term on the right side of (21) is a time shift (parallel shift) that is dependent on azimuth-frequency, and the second term performs a scaling of time. Since $D\left(f_{n}, v\right)$ is always no more than one, the change of variable is always an expansion of the data size in range time direction, and the change caused by the first term in (21) is normally much larger than the change caused by the second term.

Two problems could happen when applying the traditional Stolt mapping of (20) in FMCW SAR signal processing. First, in wide beamwidth or high squint imagery, the term $D\left(f_{n}, v\right)$ can be considerably smaller than 1 . This will greatly expand the result spectrum after Stolt mapping and hence will significantly increase the burden of calculation and the requirements for the memory size to store the result spectrum. Furthermore, the degeneration in focus quality is also reported as the increase in the value of variable change ${ }^{[19]}$. Second, the scaling in the original time variable is different for different values of $f_{n}$, which means the signal length of the range dimension after Stolt mapping is different for different azimuth positions. Therefore, the whole spectrum has to be zero padded in range to make the length of range dimension the same for all $f_{n}$ values. However, as can be seen in (6), the original range resolution is one sample after range FFT, hence it is not necessary to conduct zero padding, which will smear the peak energy of the sinc function in (5) to adjacent range bins (will be explained later), if some modifications can be made. The modified RMA takes two steps of modification to solve the problems.

The first step modifies the Stolt mapping expression. Instead of the traditional Stolt mapping used in (20), the following variable change is used

$$
\sqrt{\left(f_{0}+k t\right)^{2}-\frac{c^{2} f_{\eta}^{2}}{4 v^{2}}}=D\left(f_{\eta}, v\right) f_{0}+k t_{1}
$$

By using (22) as the Stolt mapping formula, the first term on the right side of (21) disappears, and the mapping now only corresponds to an expansion of the original time variable, which is

$$
t_{1}=\frac{t}{D\left(f_{\eta}, v\right)}
$$

This mapping removes the parallel shift of the spectrum along range dimension, which can be huge when the azimuth frequency is high. Meantime, this modified mapping still completes all the critical operations of the traditional Stolt mapping except the azimuth compression. After the modified Stolt mapping and range FFT, the azimuth signal needs to be compressed in the equivalent 'range Doppler domain' (range-frequency azimuth-frequency).

The second step to of modifying RMA is to limit the range of the new time variable. As indicated by (5) and (6) in Section 2, the range resolution of the original signal is 1 sample independent of the range sampling frequency $f_{s}$. In Stolt mapping, because the signal in zero azimuth-frequency bin does not change $(D(0, v)=1)$, the time interval between two adjacent samples after Stolt mapping in other azimuth bins should be the same as the interval in zero azimuth-frequency bin, which is $1 / f_{s}$. Therefore, the total samples in range after Stolt mapping would be

$$
N_{t}=T_{t} f_{s}=\left(\frac{f_{0}}{k}\left(D\left(f_{\eta_{\max }}, v\right)-1\right)-\frac{T}{2} \frac{D\left(f_{\eta_{\max }}, v\right)+1}{D\left(f_{\eta_{\max }}, v\right)}\right) f_{s}
$$

in traditional Stolt mapping or

$$
N_{c}=T_{c} f_{s}=\frac{T}{D\left(f_{\eta_{\max }}, v\right)} f_{s}
$$


wider than 1 sample because of the zero padding

in the case of using (25), where $\mathrm{T}$ is defined by Figure 1.

First, the situation when using (22) to perform the Stolt mapping is considered. Substitute (22) into (19) and use the approximation (23) in the range envelope, we have

$S_{11}\left(t_{1}, f_{\eta}\right)=\sigma\left(R_{0}, \eta_{c}\right) \operatorname{rect}\left(\frac{t_{1}}{T / D_{m}}\right) \operatorname{rect}_{a}\left(\frac{f_{\eta}}{B w_{a}}\right)$

$\exp \left(j \frac{4 \pi \Delta R}{c} k t_{1}+j \frac{4 \pi \Delta R}{c} D f_{0}-j 2 \pi f_{\eta} \eta_{c}\right)$

where $D\left(f_{n}, v\right)$ is replaced by $D$ and $D\left(f_{n \max }, v\right)$ is replaced by $D_{m}$ for simplicity. The length of the range envelop is $T / D_{m}$ because the signal is zero padded to length $T / D_{m}$ at all azimuth frequency bins. After range FFT, we have

$$
\begin{aligned}
& S_{12}\left(f_{1}, f_{\eta}\right)=A\left(R_{0}, \eta_{c}\right) \operatorname{sinc}\left(\frac{T}{D_{m}}\left(f_{1}-\frac{2 \Delta R}{c} k\right)\right) \\
& \operatorname{rect}_{a}\left(\frac{f_{\eta}}{B w_{a}}\right) \exp \left(j \frac{4 \pi \Delta R}{c} D f_{0}-j 2 \pi f_{\eta} \eta_{c}\right)
\end{aligned}
$$

where all the coefficient is absorbed by $A\left(R_{0}, \eta_{c}\right)$. Following the steps from (5) to (6), the $3 \mathrm{~dB}$ width of the peak of (27) is

$$
P_{1}=D_{m} \frac{T_{c}}{T}>1 \quad \text { samples }
$$

As expected, the width of the peak of the sinc function is

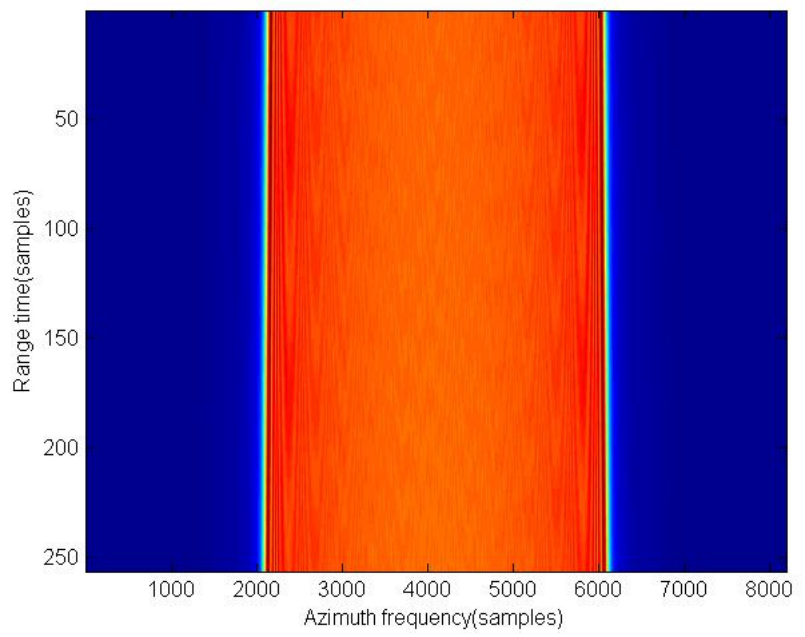

a (corresponds to an interpolation) before range FFT. However, the zero padding is not necessary because the original range resolution is only 1 sample. Therefore, there is no need to widen the peak. In the case of wide beamwidth, $T_{c}$ can be many times bigger than $T$, and hence the peak of (27) will occupy many samples in range frequency after range FFT. The negative effect of widening the peak in range is that the energy of the peak is smeared along the range.

Using the same analysis steps for the traditional Stolt mapping shows the same result as in (28). Therefore, it is not necessary to imply the complete Stolt mapping even in (23) for expanding the range variable to $\left\lceil-T / 2 D\left(f_{n}, v\right), T / 2 D\left(f_{n}, v\right)\right\rceil$. The range of the new variable can be the same as that of the old time variable for all $D\left(f_{n}, v\right)$, which is

$$
-\frac{T}{2} \leq t_{1} \leq \frac{T}{2}
$$

For the azimuth bin in which $D=0.9$, the length of the Stolt mapped signal by using both (23) and (29) will be $10 \%$ shorter than that mapped by only using (23). At the same time, the result still keeps the original range resolution and the energy is more concentrated after range FFT.

An example of the spectrums after different Stolt mapping methods are shown in Figure 2. The SAR parameters are shown in Table $\mathbf{1 .}$

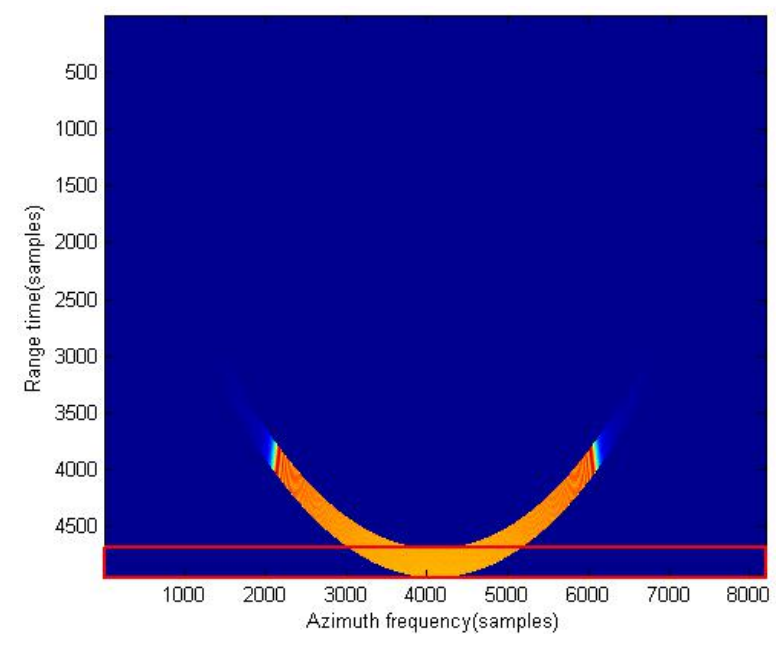

b 


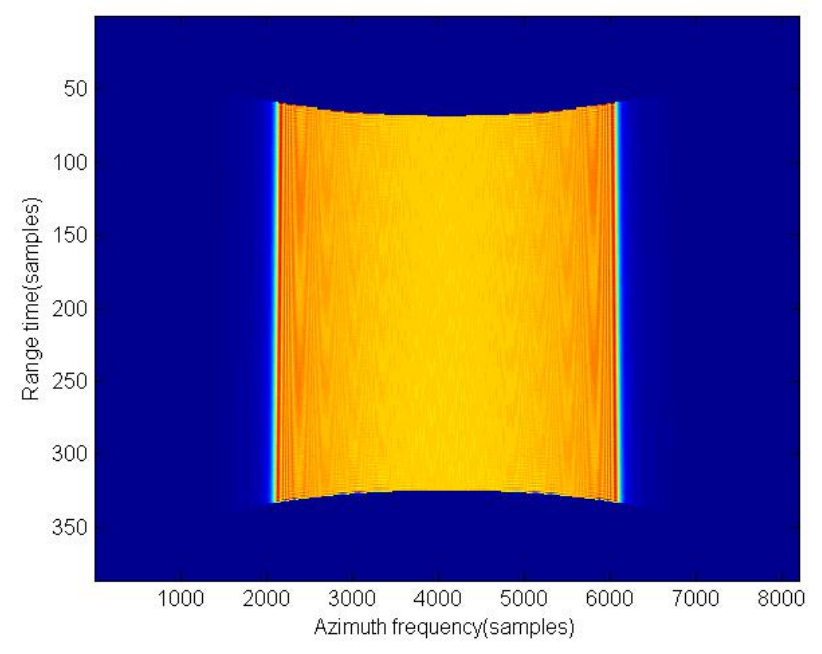

c

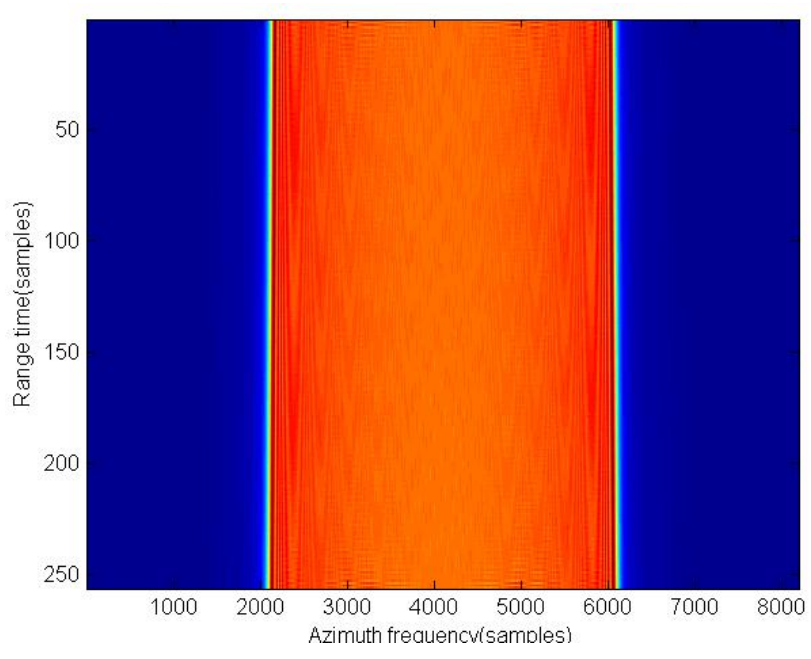

d

Figure 2. Flow diagrams for RMA and RDA.

a) Original spectrum; b) Spectrum after traditional Stolt mapping; c) Spectrum using only the first step of the modified Stolt mapping (eq. (23)); d) Spectrum using the full modified Stolt mapping (eq.(23) and (29))

Figure $2 \mathrm{a}$ shows the original 'two-dimensional spectrum' before Stolt mapping, which is 256 samples (range) by 8192 samples (azimuth). Figure 2b shows the spectrum after the traditional Stolt mapping, which expands the spectrum to 5000 samples (range) by 8192 samples (azimuth). Figure 2c is the spectrum using only the first step of the modified Stolt mapping. As can be seen, after removing the parallel shift of the time variable, the Stolt mapping only corresponds to a scaling of the variable, and the size of the result spectrum has been greatly reduced to approximately 400 samples (range) by 8192 samples (azimuth). Figure 2d is the result spectrum of the modified Stolt mapping. It has the same size as the original spectrum. Therefore, after applying the two changes to the traditional Stolt mapping, the resulted spectrum size will be minimal after Stolt mapping without losing the range resolution. Moreover, the peak energy will be still concentrated in one sample after range FFT.

\subsubsection{Azimuth compression}

After modified Stolt mapping, the expression for the result signal is

$$
\begin{aligned}
S_{2}\left(t_{1}, f_{\eta}\right)= & \sigma\left(R_{0}, \eta_{c}\right) \operatorname{rect}\left(\frac{t_{1}}{T}\right) \operatorname{rect}_{a}\left(\frac{f_{\eta}}{k_{a} T_{a}}\right) \\
& \cdot \exp \left(j \frac{4 \pi\left(R_{0}-R_{r e f}\right)}{c}\left(D\left(f_{\eta}, v\right) f_{0}+k t_{1}\right)-j 2 \pi f_{\eta} \eta_{c}\right)
\end{aligned}
$$

in which the range-azimuth coupling has been removed.
The range FT is then applied and we have

$$
\begin{aligned}
S_{3}\left(f_{1}, f_{\eta}\right) & =\int S_{1}\left(t_{1}, f_{\eta}\right) \exp \left(-j 2 \pi f_{1} t_{1}\right) d t_{1} \\
& =T \sigma\left(R_{0}, \eta_{c}\right) \operatorname{sinc}\left[T\left(f_{1}-\frac{2 k\left(R_{0}-R_{r e f}\right)}{c}\right)\right] \cdot \operatorname{rect}_{a}\left(\frac{f_{\eta}}{k_{a} T_{a}}\right) \\
& \cdot \exp \left(j \frac{4 \pi\left(R_{0}-R_{r e f}\right)}{c} D\left(f_{\eta}, v\right) f_{0}\right) \exp \left(-j 2 \pi f_{\eta} \eta_{c}\right)
\end{aligned}
$$

where $f_{1}$ is the new range frequency variable. As implied by the sinc function in (31), the range signal has been focused and the RCM has been removed. The next step is to multiply the azimuth matched filter to perform the azimuth compression, which is

$\exp \left(j \Phi_{2}\left(f_{1}, f_{\eta}\right)\right)=\exp \left(-j \frac{4 \pi\left(R_{0}-R_{r e f}\right)}{c} D\left(f_{\eta}, v\right) f_{0}\right)$

Then we have

$$
\begin{aligned}
S_{4}\left(f_{1}, f_{\eta}\right)= & T \sigma\left(R_{0}, \eta_{c}\right) \operatorname{sinc}\left[T\left(f_{1}-\frac{2 k\left(R_{0}-R_{r e f}\right)}{c}\right)\right] \\
& \cdot \operatorname{rect}_{a}\left(\frac{f_{\eta}}{k_{a} T_{a}}\right) \exp \left(-j 2 \pi f_{\eta} \eta_{c}\right)
\end{aligned}
$$

\subsubsection{Azimuth inverse Fourier transform}

An azimuth IFT (inverse Fourier transform) then generates the focused image, which is 


$$
\begin{aligned}
s\left(f_{1}, \eta\right) & =\frac{1}{2 \pi} \int S_{4}\left(f_{1}, f_{\eta}\right) \exp \left(j 2 \pi f_{\eta} \eta\right) d \eta \\
& =A \sigma\left(R_{0}, \eta_{c}\right) \operatorname{sinc}\left[T\left(f_{1}-\frac{2 k\left(R_{0}-R_{r e f}\right)}{c}\right)\right] \operatorname{sinc}\left[k_{a} T_{a}\left(\eta-\eta_{c}\right)\right]
\end{aligned}
$$

where $A$ is a constant amplitude. The amplitude of the target point is now a two dimensional sinc function and the resolutions are determined by signal fast time

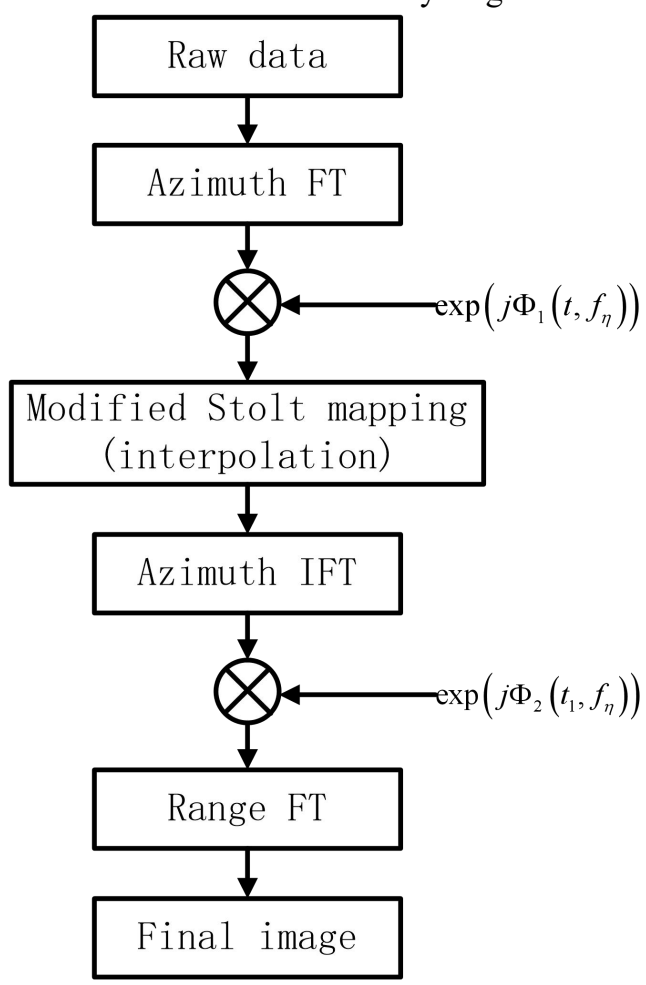

a duration $T$ in range and azimuth bandwidth $k_{a} T_{a}$ in azimuth.

\section{Comparison with the RDA}

The flow diagrams for RMA and RDA when processing FMCW SAR signals are shown in Figure 2.

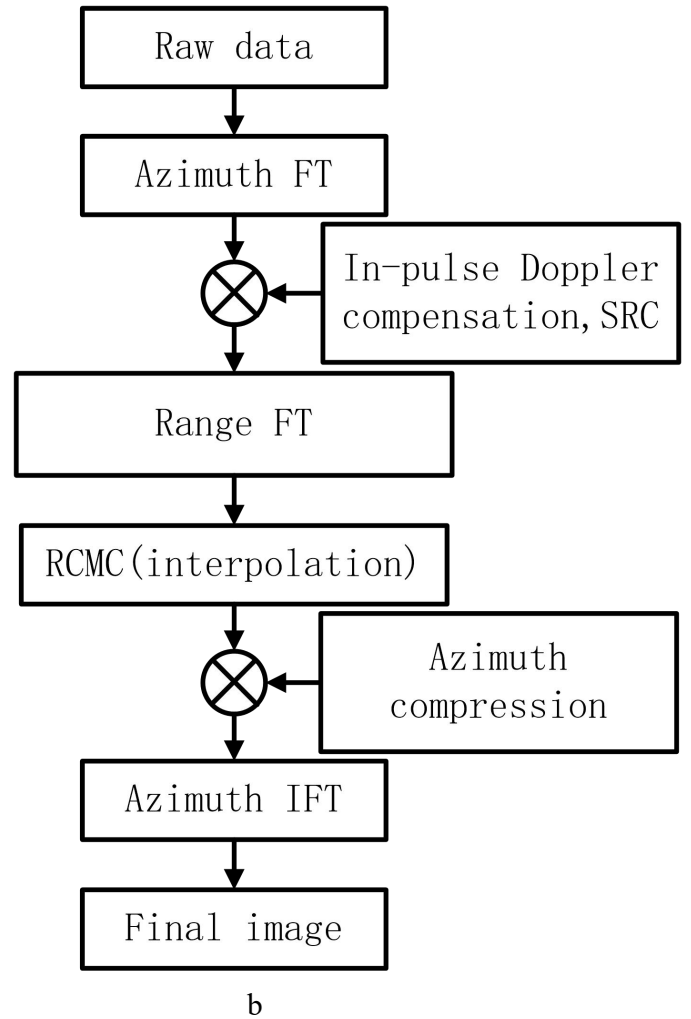

Figure 3. Flow diagrams for RMA and RDA.

a) modified RMA; b) RDA

Figure 3a shows the flow chart of the RMA. The first phase multiplication finishes the in-pulse Doppler effect removal and the RFM. The Second phase multiplication completes the azimuth compression. One range $\mathrm{FT}$, one azimuth $\mathrm{FT}$, one azimuth IFT and one interpolation operation for Stolt mapping are needed for the signal processing.

Figure $3 \mathrm{~b}$ shows the flow diagram of the RDA. The first phase multiplication finishes the in-pulse Doppler effect removal and the approximated SRC. The other options of applying the SRC can be found in ${ }^{[18]}$. The Second phase multiplication completes the azimuth compression. One range FT, one azimuth FT, one azimuth IFT and one interpolation operation for RCMC are needed for the processing.

As can be seen from Figure 3, the modified RMA has roughly the same computational load and the same memory requirements as the RDA if the same orders of interpolation are used. This is because the modified RMA does not expand the spectrum in range direction when performing the Stolt mapping. The RMA will generate a better image than RDA because it does not make any approximations during the image processing, while the RDA only approximately compensates the SRC and totally neglects the higher order terms of range-azimuth coupling.

\section{Results}

This section first uses simulation and then uses real data to verify modified RMA.

\subsection{Simulation}


The parameters for simulation are shown in Table 1. Figure 4 shows the parallel shift (21).

\begin{tabular}{|c|c|c|}
\hline Parameter & Value & Units \\
\hline SAR speed & 50 & $\mathrm{~m} / \mathrm{s}$ \\
\hline PRF (Pulse repetition frequency) & 200 & $\mathrm{~Hz}$ \\
\hline Squint angle & 0 & $\mathrm{rad}$ \\
\hline Centre frequency & 400 & $\mathrm{MHz}$ \\
\hline Signal bandwidth & 7.5 & $\mathrm{MHz}$ \\
\hline Target distance & 2000 & $\mathrm{~m}$ \\
\hline Scene centre & 2560 & $\mathrm{~m}$ \\
\hline Antenna beamwidth & 42.97 & degree \\
\hline Data azimuth length & 8192 & samples \\
\hline Data range length & 256 & samples \\
\hline
\end{tabular}

Table 1. Simulation parameters

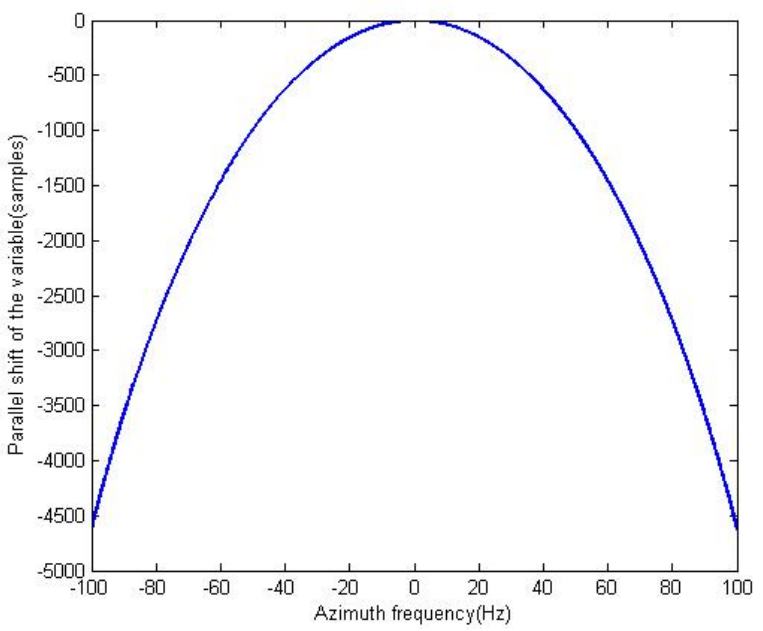

Figure 4. Parallel shift of the variable in Stolt mapping.

As shown by Figure 4, at both edges of the azimuth aperture, the parallel shift caused by the first term of (21) is more than 4500 samples, which means the length of the range spectrum should be greatly expanded to store the whole result of Stolt mapping. This corresponds to a significant expansion in the size of the signal spectrum (see Figure 2b) because the length of the original spectrum before Stolt mapping is only 256 samples in range. The modified variable substitution avoids this parallel shifting, which greatly reduces the computational load and required memory size for performing the Stolt mapping (see Figure 2c).

$\left(f_{0}\left(D\left(f_{n}, v\right)-1\right) / k\right)$ of the variable in the traditional Stolt mapping, which corresponds to the first term of 
Figure 6a is the range FFT result of the traditional Stolt mapped spectrum. As expected, the RCM has been removed. However, the range width of the azimuth trajectory is wide because of the zero padding (due to Stolt mapping) before FFT. As can be seen, the energy is smeared in range. Figure $\mathbf{6 b}$ is the range FFT after the modified Stolt mapping. The RCM has been corrected and the energy concentrates in one range bin. The range resolution is the same as the original signal.

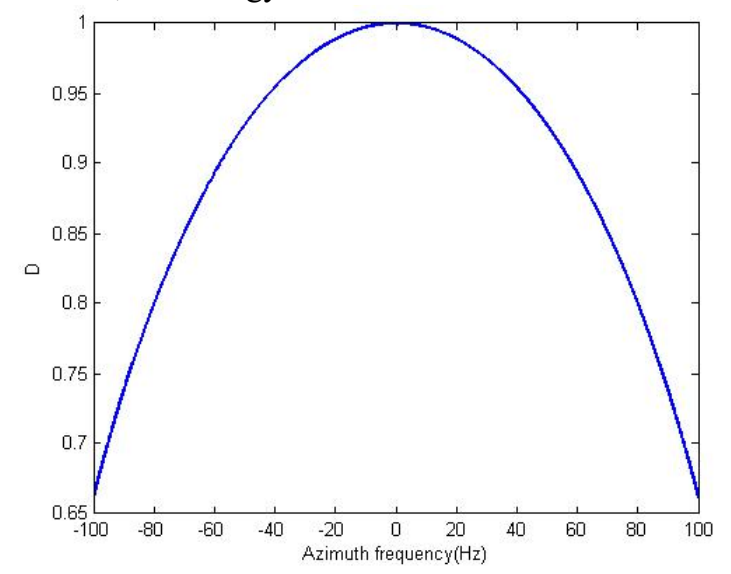

Figure 5. $D\left(f_{n}, v\right)$ defined by (17).

a) Using traditional Stolt mapping; b) Using modified Stolt mapping

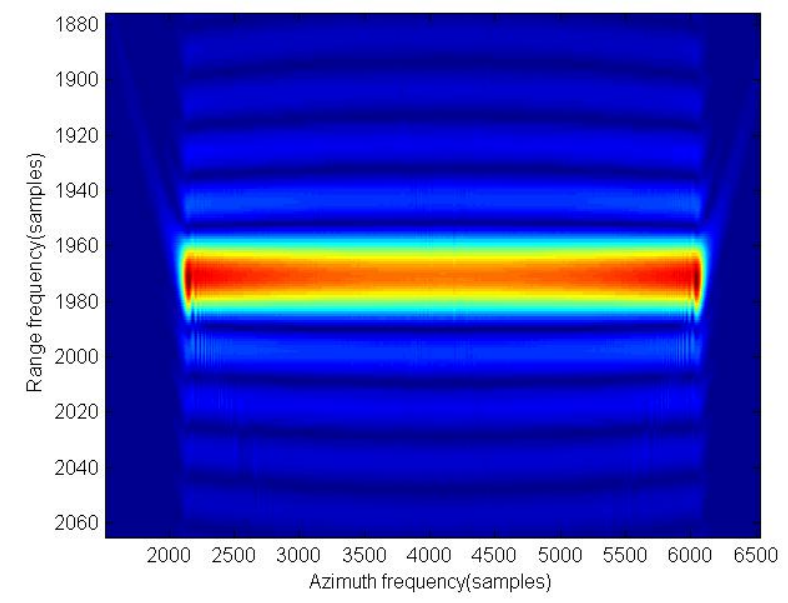

a

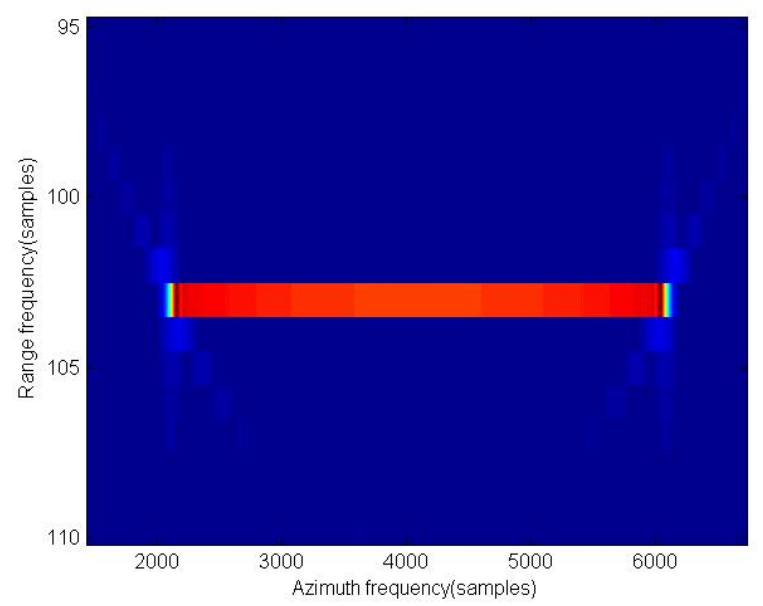

b

Figure 6. Range FFT of the Stolt mapped spectrum.

One method of using the spectrum (Figure 2b) after the traditional Stolt mapping is given in [17] (page 408). This method cuts a rectangular area in the result spectrum for imaging. However, when the Stolt mapping significantly skews the original spectrum due to large beamwidth or high squint angle, the rectangular area that can be used for imaging is very small. In this case, the range resolution will be decreased.

The way of keeping the range resolution is to maintain the range length of the spectrum after traditional Stolt mapping the same as the original spectrum, which corresponds to truncate the first 256 range samples (original spectrum is 256 samples in range). The truncated spectrum after traditional Stolt mapping and the spectrum after modified Stolt mapping is shown in Figure 7.

Figure $7 \mathrm{a}$ is the result of the modified Stotl mapping, which is the same as what is shown in Figure $2 d$. Figure $7 \mathrm{~b}$ is the result spectrum (256 samples by 8192 samples) cut from the bottom of Figure $2 b$ (the part in the red rectangular in Figure 2b).

Figure 8 shows the compression results of the traditional RMA and the modified RMA by using the spectrums in Figure $7 \mathrm{a}$ and Figure $7 \mathrm{~b}$. Interpolation is used to reveal the sidelobes more clearly. In Figure 8a, the point target is well focused by using the proposed RMA. In Figure 8b, the target is defocused, and the azimuth mainlobe is wider than the range 


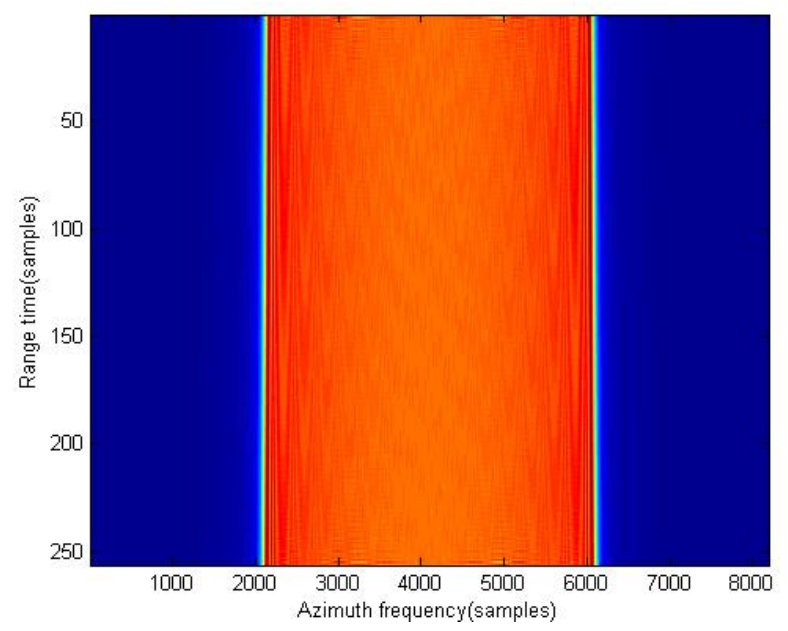

a

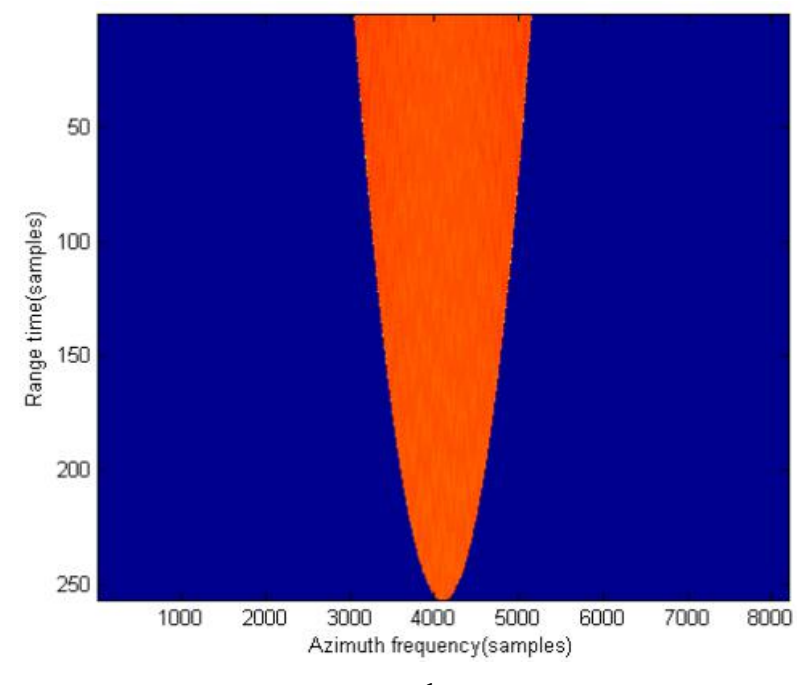

$\mathrm{b}$

Figure 7. Original spectrum and the result spectrums after Stolt mapping.

a) Result of the modified Stolt mapping; b) Truncated spectrum of the traditional Stolt mapping result

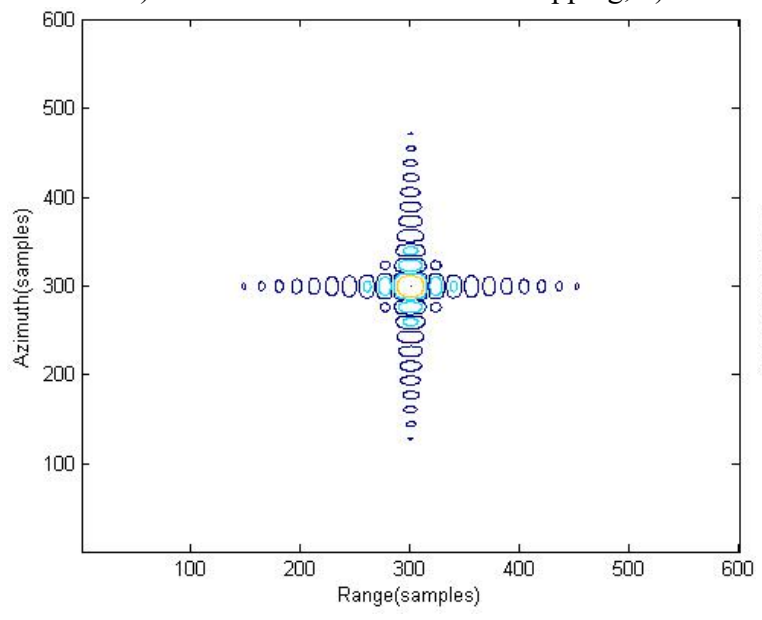

a

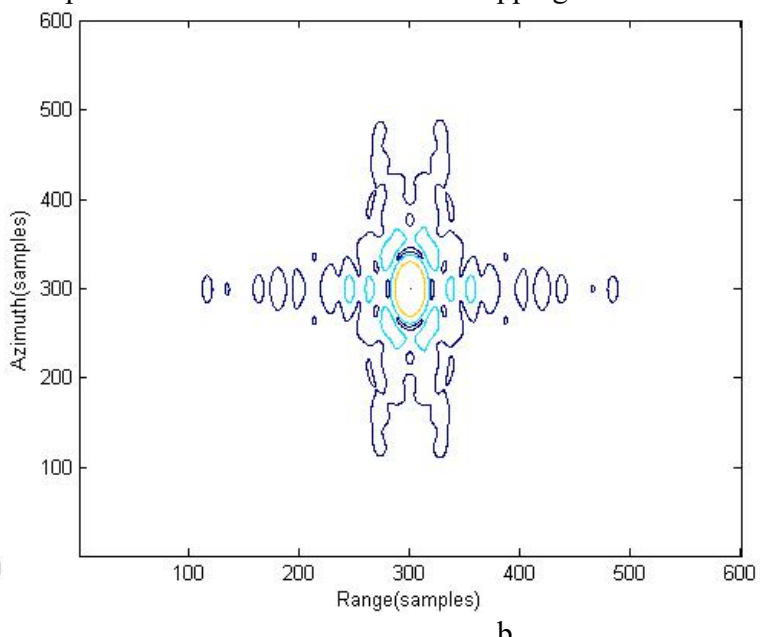

Figure 8. Compression results by using the modified RMA and the traditional RMA by using the same size of spectrum. a) Proposed RMA;b) Traditional RMA 


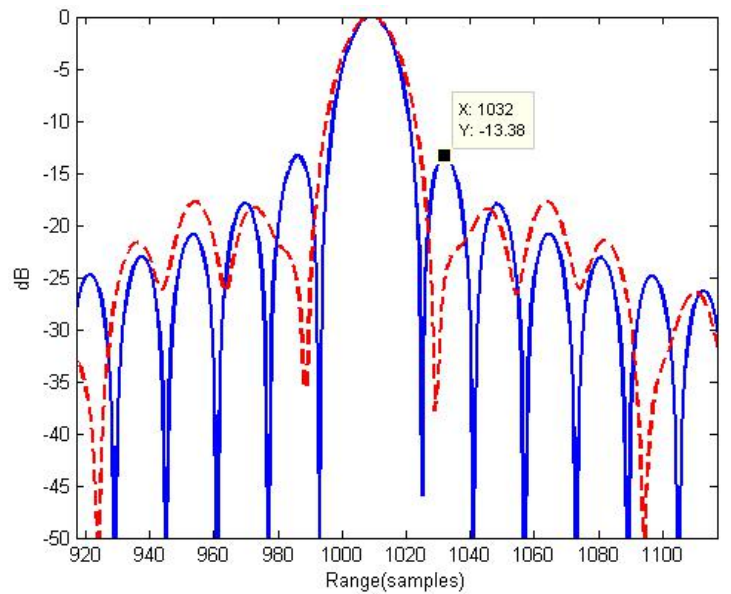

a

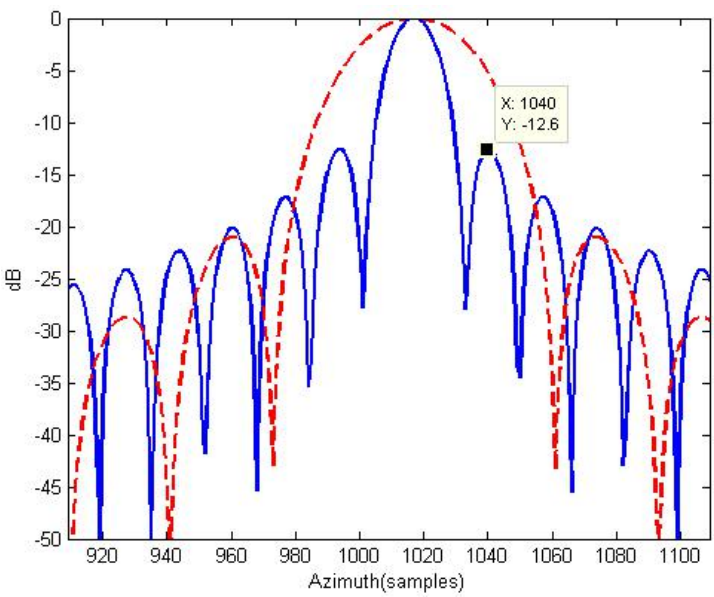

Figure 9. Comparison of the range and azimuth compression quality focused target.

a) Range slices;b) Azimuth slices

Figure 9 shows the comparison for the compression quality of the focused target when using the traditional RMA and the proposed RMA separately. The solid lines are the range and azimuth slices from Figure $\mathbf{8 a}$, while the dashed lines are from Figure $\mathbf{8 b}$. For the solid line in Figure 9a and Figure $9 \mathrm{~b}$, the $3 \mathrm{~dB}$ width of the mainlobes are all 15 samples, meaning the range and azimuth resolution are the same. The mainlobe of the dashed line in Figure $9 \mathrm{~b}$ is significantly wider than that of the solid line because the azimuth bandwidth is greatly decreased due to the truncate of the result after traditional Stolt mapping when using the same size of the spectrum.

\subsection{Real data processing}

The real data was collected by an FMCW SAR. The radar is composed by several customized printed circuit boards and some off-the-shelf microwave components. The radar works at $\mathrm{C}$ band and has a maximum bandwidth of $150 \mathrm{MHz}$. The data used in this section was collected during a ground moving vehicle test. The antenna configuration in the test is shown in Figure 10.

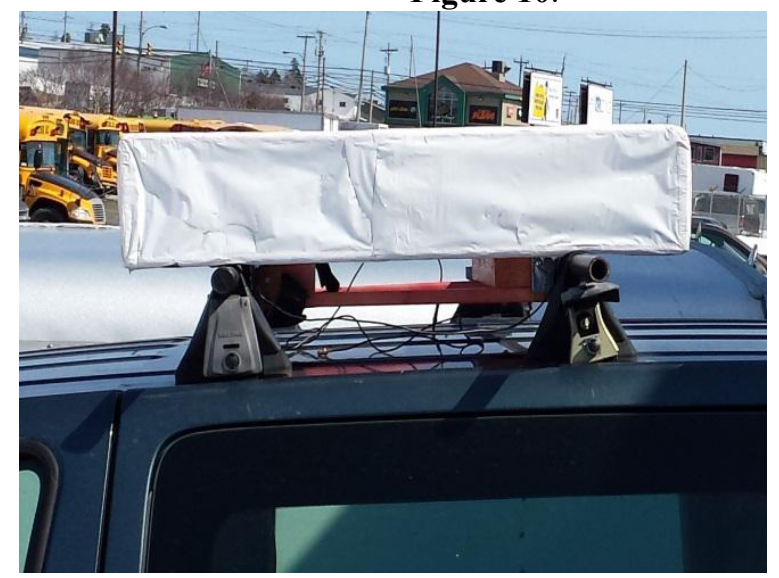

Figure 10. The antenna configuration in the ground test. 


\begin{tabular}{|c|c|c|}
\hline Parameter & Value & Units \\
\hline SAR speed & 60 & $\mathrm{~km} / \mathrm{h}$ \\
\hline Bandwidth & 150 & $\mathrm{MHz}$ \\
\hline Centre frequency & 5590 & $\mathrm{MHz}$ \\
\hline PRF & 250 & $\mathrm{~Hz}$ \\
\hline Squint angle & 0 & degree \\
\hline Antenna azimuth beamwidth & 8 & degree \\
\hline
\end{tabular}

Table 2. Real data parameters

Two $2 \times 8$ patch array antennas for transmitting and receiving are enclosed in the white box in Figure 1. The antennas were mounted on the top of the van by a wood frame; the radar was mounted inside the van and connected to the antennas via coaxial cables.

The parameters used in the ground test is shown in Table 2. The images obtained by the traditional RMA and the modified RMA are shown in Figure 11. Vertical direction is range and horizontal direction is azimuth.

The images obtained by the traditional RMA and the modified RMA are shown in Figure 11. Four orders of interpolation are used to perform the Stolt mapping. No window is applied in either the range direction or the azimuth direction FFT for better comparison of the focusing quality because noise is not eliminated by windowing at all and will be more obvious in the final images. The same size of spectrum after Stolt mapping are used to generate Figure 11a and Figure $11 \mathrm{~b}$ as has been shown in the simulation in Figure 7.

As shown by Figure 11, the image generated by the modified RMA has a lower level noise than the one generated by the traditional RMA. This is more clearly shown by the area marked by the red rectangular in Fig. 11a. By comparing the areas in the red box of Figure 11a with the same area of Figure 11b, a higher background noise is observed in Figure 11a.

In Figure 12, the range compression quality is compared between the traditional and the proposed RMA. The solid line is the range slice of the marked point in Figure 11a while the dashed line is that of the same point in Figure 11b. An improvement in the range focusing quality (the dashed line has narrower mainlobe) of the proposed RMA than the traditional RMA can be observed. The improvements using the modified RMA are not as significant as in Figure 8 because the SAR parameters used in the field test are very normal (narrow beamwidth, no squint). While the parameters used in simulation are very extreme in order to reveal the improvement of the proposed RMA. 


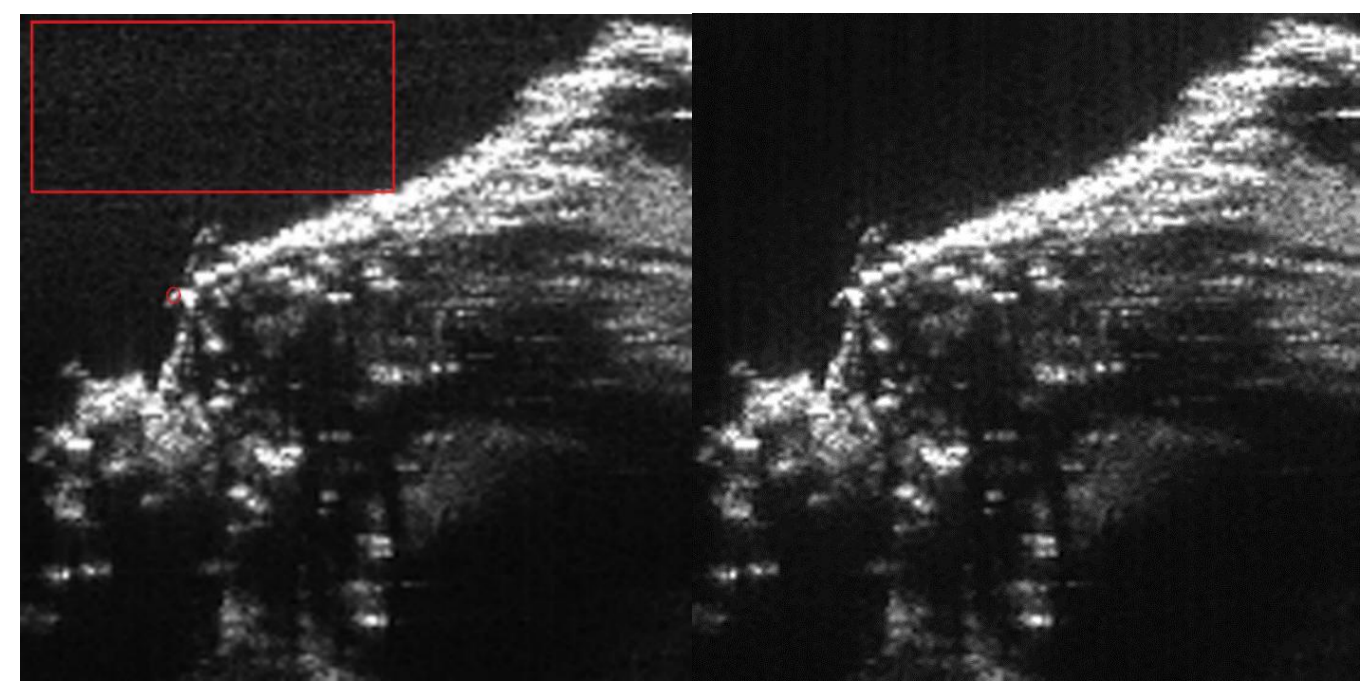

a

b

Figure 11. Images processed by different RMAs. a) Traditional RMA; b) Proposed RMA.

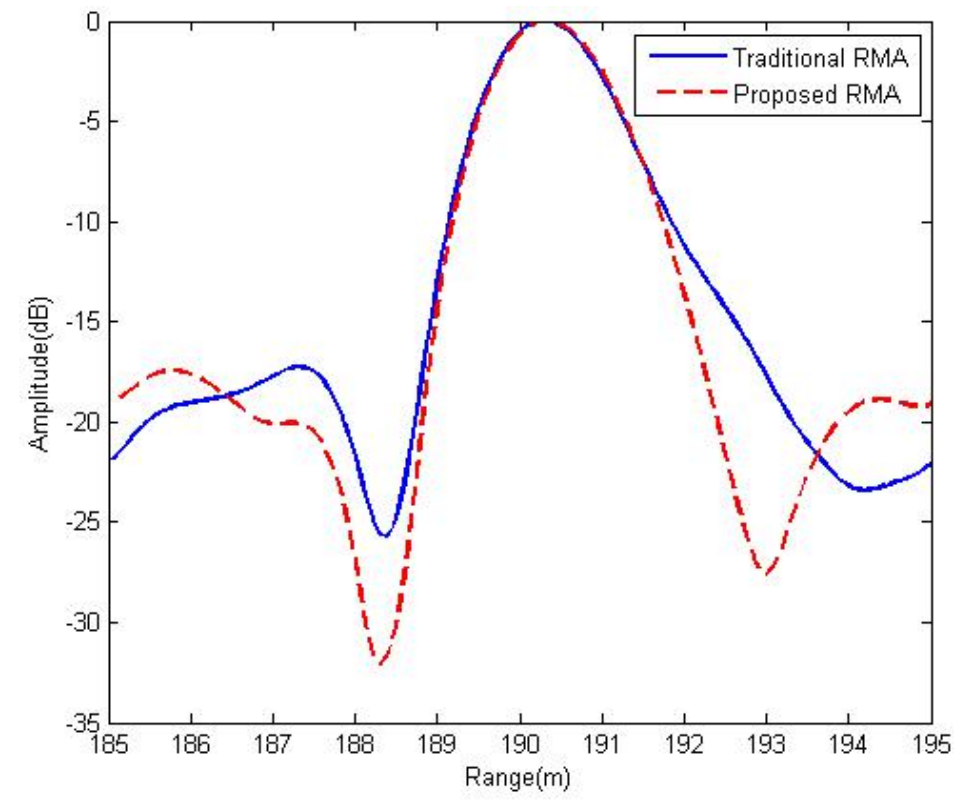

Figure 12. Comparison of the range profiles of an isolated strong point target marked by the red circle in Figure 11a.

\section{Conclusion}

A modified RMA is proposed and used to process the FMCW SAR signal. The modified RMA takes the advantage of the special characteristics of the IF signal in dechirp-on-receive FMCW radar systems to decreases the computational load and the memory required during image generation. The modified RMA now have almost the same computational speed as compared to the RDA if the same orders of interpolation are used. A better focusing quality is also obtained in the proposed RMA than in the traditional RMA when using the same size of the spectrum after Stolt mapping. The effectiveness of the proposed algorithm is verified by both simulation and real data at the end.

\section{Author contributions}

Yake Li designed and built the FMCW radar used in the result section of this paper, conducted the SAR experiment and proposed the signal processing method introduced in this paper.

Siu O'Young supervised the research work and provided helpful suggestions.

\section{References}

1. Edrich, M. 'Design Overview and Flight Test Results of the Miniaturised SAR Sensor MISAR'. Proc. EuRAD, Amsterdam, Netherlands, Oct. 2004.

2. Edrich, M. 'Ultra-lightweight synthetic aperture radar based on a $35 \mathrm{GHz}$ FMCW sensor concept and online raw data transmission', IEE Pro. Radar and Sonar Navig. 2006, 153, pp. 129-134.

3. Berizzi, F., Martorella, M., Cacciamano, A., Capria, 
A,: 'A Contrast-Based Algorithm for Synthetic Range-Profile Motion Compensation', IEEE Trans. Geosci. Remote Sens. 2008, 46, pp. 3053-3062.

4. Essen, H., Stanko, S., Sommer R., Johannes, W., Wahlen, A., Wilcke, J., Hantscher, S.: 'Millimetre Wave SAR for UAV Operation. Proc. Asia-Pacific Microwave Conference', Melbourne, Australia, 2011.

5. Max, F.; Wahlen, A.; Peter, W.; Erich, M. Processing of MIRANDA35 FMCW-SAR Data using a Time-Domain Algorithm. Proc. EUSAR, Berlin, Germany, 2014.

6. Rossum, W. V., Otten, M., Dorp, P. V.: 'Multichannel FMCW SAR', Proc. EUSAR, Nuremberg, Germany, Apr. 2012.

7. Edwards, M., Madsen, D., Stringham, C., Margulis, A., Wicks, B., Long, D.: 'microASAR: A Small, Robust LFM-CW SAR for Operation on UAVs and Small Aircraft', Proc. IGASS 2008.

8. Wit, J. J. M. de, Meta, A., Hoogeboom, P.: 'Modified Range-Doppler Processing for FM-CW Synthetic Aperture Radar', IEEE Geosci. Remote Sens. Lett., 2006, 3, pp. 83-87.

9. Meta, A., Hoogeboom, P., Ligthart, L. P.: 'Signal Processing for FMCW SAR', IEEE Trans. Geosci. Remote Sens., 2007, 45, pp. 3519-3532.

10. Zaugg, E. C., Long, D.: 'Generalized Frequency Scaling and Backprojection for LFM-CW SAR Processing', IEEE Trans. Geosci. Remote Sens., 2015, 53, pp. 3600-3614.

11. Wang, R., Loffeld, O., Nies, H., Knedlik, S., Hagelen, M., Essen, H.: 'Focus FMCW SAR Data Using the Wavenumber Domain Algorithm', IEEE Trans. Geosci. Remote Sens., 2010, 48, pp.
2109-2118.

12. Cafforio, C., Prati, C., Rocca, F.: 'SAR data focusing using seismic migration techniques', IEEE Trans. on Aerospace and Electr. Syst., 1991, 27, pp. 194-207.

13. Cafforio, C., Prati, C., Rocca, F.: 'Full resolution focusing of Seasat SAR images in the frequency-wave number domain', Int. Journal of Remote Sens., 1991, 12, pp. 491-510.

14. Reigber, A., Alivizatos, E., Potsis, A., Moreira, A.: 'Extended wavenumber-domain synthetic aperture radar focusing with integrated motion compensation', IET Radar Sonar Navig., 2006, 153, pp. 301-310.

15. Zhang, L., Sheng, J., Xing, M., Qiao, Z., Xiong, T., Bao, Z.: 'Wavenumber-Domain Autofocusing for Highly Squinted UAV SAR Imagery', IEEE Sensors Journal, 2012, 12, pp. 1574-1588.

16. Xu, G., Xing, M., Zhang, L., Bao, Z.: 'Robust Autofocusing Approach for Highly Squinted SAR Imagery Using the Extended Wavenumber Algorithm', IEEE Trans. Geosci. Remote Sens., 2013, 51, pp. 5031-5046.

17. Carrara, W. G., Goodman, R. S. and Majewski, R. M.: 'Spotlight Synthetic Aperture Radar: Signal Processing Algorithms', Artech House, 1995

18. Cumming, I. G., Wong, F. H.: 'Digital processing of synthetic aperture radar', Artech House MA, USA, 2005.

19. Vandewal, M.; Speck, R.; Helmut: 'Efficient and Precise Processing for Squinted Spotlight SAR through a Modified Stolt Mapping', EURASIP Journal on Advances in Signal Processing., 2007, pp.1-7. 